\title{
NGHIÊN CÚU, THIẾT KẾ VÀ CHẾ TẠO SIÊU VÂT LIỆU KHÔNG PHỤ THUỘC VÀO PHÂN CỰC SÓNG ĐIỆN TÙ
}

\author{
Nguyễn Thị Hiền ${ }^{1,2, ~ *, ~ V u ̃ ~ Đ i ̀ n h ~ Q u i ́ ~}{ }^{1}$, Trịnh Thị Giang ${ }^{1}$, Nguyễn Thanh Tùng ${ }^{1}$, \\ Vũ Đình Lãm ${ }^{1}$
}

${ }^{I}$ Viện Khoa học vật liệu, Viện Hàn lâm Khoa học và Công nghệ Việt Nam, 18 Hoàng Quốc Việt, Cầu Giấy, Hà Nội

${ }^{2}$ Truờng Đại học Khoa học, Đại học Thái Nguyên, Phwờng Tân Thịnh, Tp. Thái Nguyên

*Email:ncshien@gmail.com

Đến Tòa soạn: 11/7/2015; Chấp nhận đăng: 28/11/2015

\section{TÓM TẮT}

Gần đây, để thu được vật liệu có độ từ thẩm âm và vật liệu chiết suất âm các nhà nghiên cứu thường sử dụng siêu vật liệu có cấu trúc dạng vòng cộng hưởng có rãnh (split-ring resonator - SRR) hay cặp thanh kim loại (cut-wire-pair - CWP) kết hợp với các lưới dây kim loại liên tục. Tuy nhiên, hạn chế của việc sử dụng các cấu trúc này là phụ thuộc rất mạnh vào phân cực của sóng điện từ chiếu đến. Trong báo cáo này, chúng tôi sử dụng cấu trúc biến đổi của $\mathrm{CWP}$ - cấu trúc cặp đĩa có tính đối xứng cao để hạn chế nhược điểm trên. Ô cơ sở của cấu trúc này gồm một cặp đĩa kim loại cách nhau bởi một lớp điện môi,tương tác với từ trường ngoài tạo ra độ từ thẩm âm $(\mu<0)$. Bằng cách mở rộng bán kính của đĩa cho đến khi các đĩa chạm vào nhau, tính chiết suất âm có thể thu được $(\mathrm{n}<0)$. Kết quả này mở ra khả năng có thể điều chỉnh tính chất của vật liệu bằng tác động ngoại vi khi thay thế kim loại bằng vật liệu thích hợp. Cấu trúc tối ưu tạo ra chiết suất âm không phụ thuộc vào phân cực của sóng tới tìm kiếm được gồm các đĩa liên tiếp chạm vào nhau dọc theo cả hai trục $\mathrm{x}(\mathbf{H})$ và $\mathrm{y}(\mathbf{E})$. Kết quả này là một bước quan trọng để tiến gần đến các ứng dụng thực tế của hiện tượng chiết suất âm khi không phụ thuộc phân cực. Các kết quả nghiên cứu sử dụng mô phỏng, thực nghiệm và tính toán trong nghiên cứu trùng khớp với nhau.

Tù khóa: siêu vật liệu chiết suất âm không phụ thuộc phân cực, cấu trúc cặp đĩa, lưới đĩa.

\section{GIỚI THIỆU}

Trong tự nhiên, các tính chất vật lí của vật liệu thường được quyết định bởi tính chất của các nguyên tử và cấu trúc mạng tinh thể của những nguyên tử này [1]. Ý tưởng về sự tồn tại của những nguyên tử nhân tạo được sắp xếp trong các mạng tinh thể nhân tạo, cho phép con người có thể tạo ra những tính chất mới lạ không tồn tại trong tự nhiên, từ lâu đã thu hút sự quan tâm của các nhà khoa học. Với sự phát triển của khoa học công nghệ, những "siêu nguyên tử" có những tính chất đặc biệt, được tạo ra bằng cách tổ chức có chủ ý nhiều nguyên tử cùng hoặc 
khác loại, đã ra đời từ đầu những năm 80 của thế kỷ trước [2]. Tuy nhiên, thú vị hơn cả là sự ra đời của vật liệu điện từ nhân tạo - siêu vật liệu (Metamaterials - MMs). MMs được xây dựng dựa trên những "giả nguyên tử", là những mạch cộng hưởng điện từ nhỏ hơn nhiều lần bước sóng mà tại đó các tính chất đặc biệt của MMs xuất hiện. Bằng cách thay đổi tính chất và mạng tinh thể (quy luật sắp xếp) của các "giả nguyên tử" này một cách đồng thời, các nhà khoa học có thể thu được những tính chất bất thường không tồn tại trong vật liệu tự nhiên. Một trong những tính chất được tìm kiếm đầu tiên của MMs là khả năng tạo ra môi trường có chiết suất âm. Ý tưởng về sự tồn tại của vật liệu có chiết suất âm được đề xuất vào năm 1968 bởi Veselago [3], dựa trên sự kết hợp đồng thời của vật liệu có độ từ thẩm âm và độ điện thẩm âm trên cùng một dải tần số. Tuy nhiên, sau hơn 30 năm kể từ đề xuất của Veselago, mô hình đầu tiên về vật liệu có chiết suất âm mới được đưa ra bởi John B. Pendry vào năm 1999 [4]. Mô hình này dựa trên cấu trúc vòng cộng hưởng hở (split-ring resonator). Sau đó năm 2000, D. R. Smith và cộng sự [5] lần đầu tiên chứng minh bằng thực nghiệm sự tồn tại của vật liệu chiết suất âm.

Để khai thác các khả năng ứng dụng tính chất đặt biệt của vật liệu này, các nhà khoa học trên thế giới ngày càng tập trung nghiên cứu một cách mạnh mẽ. Mục đích hiện tại của các nhà khoa học là sớm đưa siêu vật liệu vào ứng dụng trong thực tế đối với các thiết bị hoạt động ở vùng tần số sóng điện từ. Tuy nhiên, trước khi đưa vật liệu này vào ứng dụng rộng rãi, vẫn còn tồn đọng khá nhiều vấn đề cần được giải quyết một cách thỏa đáng. Như bằng cách nào để chế tạo một cách đơn giản, dễ dàng và có tính đối xứng cao, giảm sự tiêu hao, điều khiển tính chất của vật liệu bằng các tác động ngoại vi (quang, nhiệt, điện, từ...) hay mở rộng vùng tần số hoạt động của vật liệu. Trong số đó việc tìm kiếm cấu trúc đẳng hướng không phụ thuộc vào phân cực đang là một chủ đề thu hút khá nhiều sự quan tâm của các nhà nghiên cứu $[6,7]$. Trong bài báo này, chúng tôi sử dụng cấu trúc cặp đĩa có tính đối xứng cao để tạo ra vật liệu từ có độ từ thẩm âm không phụ thuộc vào phân cực. Sau đó, bằng cách mở rộng bán kính của đĩa cho đến khi các đĩa liên tiếp chạm vào nhau, tính chiết suất âm thu được. Kết quả này đưa ra một gợi ý là có thể điều chỉnh tính chất của vật liệu bằng các tác động ngoại vi. Cấu trúc tối ưu để tạo ra vùng chiết suất âm không phụ thuộc vào phân cực của sóng tới tìm kiếm được trong nghiên cứu này gồm các đĩa liên tiếp chạm vào nhau dọc theo cả hai trục $\mathrm{x}(\mathbf{H})$ và $\mathrm{y}(\mathbf{E})$ (với hằng số mạng $a_{x}=$ $a_{y}$ ). Kết quả này là một bước quan trọng để tiến gần đến các ứng dụng thực của hiện tượng chiết suất âm khi không phụ thuộc phân cực.

\section{THIẾT KẾ, THỬC NGHIỆM VÀ MÔ PHỎNG}

Như chúng ta đã biết, có hai cấu trúc rất quen thuộc được sử dụng để tạo ra độ từ thẩm âm, đó là cấu trúc vòng cộng hưởng có rãnh (split-ring resonator - SRR) và cấu trúc cặp thanh kim loại ( cut - wire pair - CWP). Tuy nhiên, cấu trúc CWP có một số ưu điểm rõ rệt so với cấu trúc SRR truyền thống. Hình dạng của cấu trúc CWP đơn giản hơn rất nhiều so với SRR, đặc biệt với cách phân cực cho phép sóng điện từ chiếu vuông góc với mặt phẳng mẫu và từ trường chỉ cần vuông góc với mặt bên của cặp thanh kim loại nên có thể tạo ra độ từ thẩm âm chỉ với một lớp cấu trúc. Dựa trên cấu trúc CWP, với biến đổi nhỏ, cấu trúc cặp đĩa (dish pair - DP) được thiết kế và chỉ ra như trên Hình 1(a). Mục đích cải tiến của cấu trúc DP này là sử dụng tính đổi xứng của hình tròn để tạo ra sự đẳng hướng (không phụ thuộc vào phân cực) đối với sóng điện từ chiếu đến. Đặc biệt hơn, trong báo cáo này, sử dụng những ưu điểm về tính đối xứng của cấu trúc $\mathrm{DP}$, kết hợp với các dây liên tục được tạo ra đơn giản bằng cách mở rộng bán kính hình tròn cho đến khi các hình tròn liên tiếp chạm vào nhau, đề tạo ra cấu trúc lưới đĩa (dish - net $-\mathrm{DN}$ ) có chiết suất âm. Ô cơ sở của cấu trúc lưới đĩa được chỉ ra trên Hình 1(c). Ngoài ra, trên Hình 
1(a) và 1 (c) còn chỉ rõ sự phân cực của sóng điện từ chiếu đến các mẫu nhằm thu được các hiệu ứng mong muốn ban đầu.

Các mẫu có cấu trúc DP và DN được chế tạo sử dụng tấm mạch in (printed circuit board) với lớp đồng (có chiều dày $t_{m}=0,036 \mathrm{~mm}$ ) được phủ ở hai mặt, giữa là lớp điện môi có chiều dày $t_{d}=0,4 \mathrm{~mm}$, hằng số điện môi 4,1 . Hằng số mạng theo các trục $\mathrm{x}(\mathbf{H}), \mathrm{y}(\mathbf{E})$ và bán kính hình tròn lần lượt là $a_{x}=11 \mathrm{~mm}, a_{y}=8 \mathrm{~mm}, R=3 \mathrm{~mm}$. Hình $1(\mathrm{~b})$ và $2(\mathrm{~b})$ lần lượt là mẫu $\mathrm{DP}$ và $\mathrm{DN}$ chế tạo được. Các thông số lựa chọn nhằm phù hợp với phép đo phổ truyền qua với dải tần đo từ 12 - 17 GHz. Phép đo này được thực hiện trên hệ network analyzer đặt trong không khí.

Trong nghiên cứu này, phần mềm mô phỏng thương mại CST Microwave Studio được sử dụng để thiết kế và mô hình hóa tính chất của vật liệu. Nhờ đó, ta thu được các thông tin về các thông số tán xạ (truyền qua, phản xạ và pha của chúng) cũng như các đặc trưng về dòng và năng lượng. Cuối cùng, các thông số tán xạ thu được kết hợp với phương pháp tính toán của Chen [8] sẽ cho ta biết giá trị của các tham số điện từ hiệu dụng $\varepsilon, \mu$ và $n$.

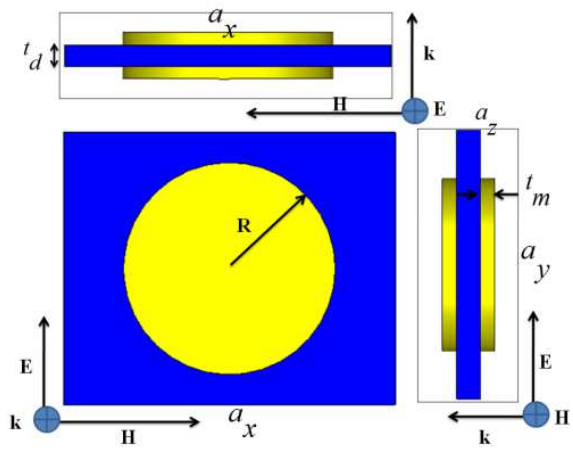

(a)

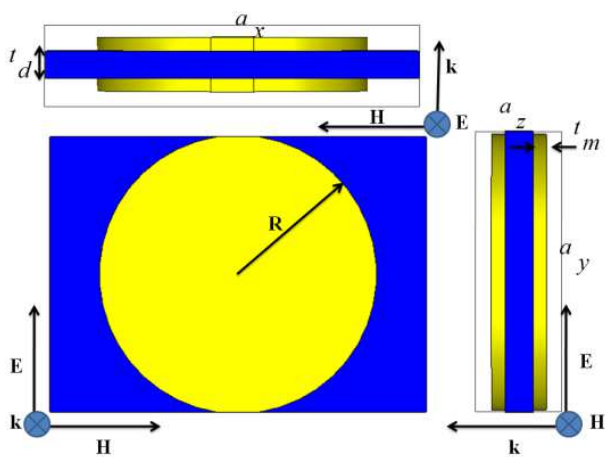

(c)

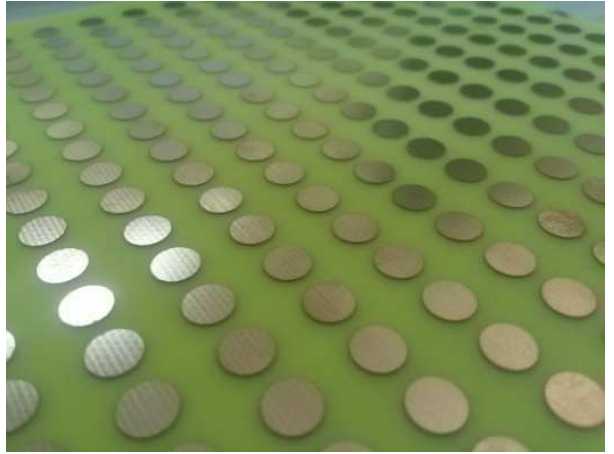

(b)

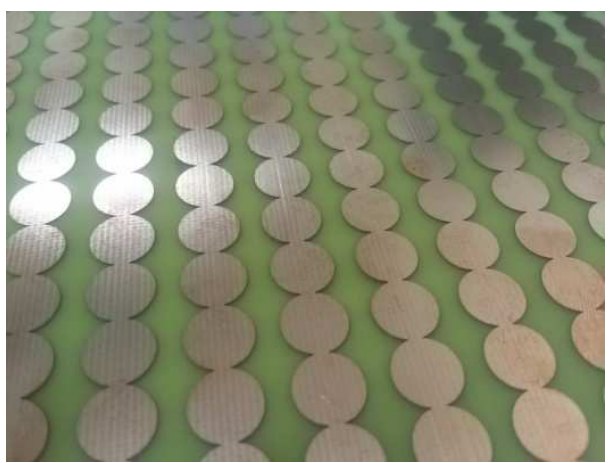

(d)

Hình 1. a) Ô cơ sở của cấu trúc cặp đĩa (DP) và sự phân cực sóng điện từ,

b) Mẫu chế tạo có cấu trúc cặp đĩa,

c) Ô cơ sở của cấu trúc lưới đĩa $(\mathrm{DN})$ và sự phân cực sóng điện từ, d) Mẫu chế tạo có cấu trúc lưới đĩa.

Các tham số cấu trúc $a_{x}=11 \mathrm{~mm}, a_{y}=8 \mathrm{~mm}, t_{d}=0,4 \mathrm{~mm}, t_{m}=0,036 \mathrm{~mm}$.

\section{KẾT QUẢ VÀ THẢO LUẬN}

\subsection{Các kết quả nghiên cứu cấu trúc cặp đĩa (dish pair - DP)}


Các kết quả nghiên cứu trước đây với cấu trúc SRR và CWP cho thấy khi chúng tương tác với sóng điện từ đều xảy ra hai cộng hưởng khác nhau: cộng hưởng từ và cộng hưởng điện. Cộng hưởng điện tạo ra độ điện thẩm âm $(\varepsilon<0)$, cộng hưởng từ tạo ra độ từ thẩm âm $(\mu<0)$. Do DP là một cấu trúc biến đổi của CWP nên hoàn toàn có khả năng thu được các vùng có độ từ thẩm âm và điện thẩm âm khi tương tác với sóng điện từ. Tuy nhiên, do việc tạo ra vùng có độ từ thẩm âm còn rất nhiều hạn chế và khó khăn so với việc tạo ra vùng có độ điện thẩm âm nên nó được quan tâm nghiên cứu nhiều hơn. Cũng chính vì lí do đó nên trong bài báo này, chúng tôi tập trung đi sâu nghiên cứu vùng có độ từ thẩm âm của cấu trúc DP trong dải tần số mô phỏng, chế tạo và đo đạc $(12 \mathrm{GHz}-18 \mathrm{GHz})$ nhằm mục đích tiến tới việc tạo ra vật liệu chiết suất âm không phụ thuộc vào phân cực. Trên Hình 2(a) là kết quả mô phỏng và thực nghiệm phổ truyền qua của cấu trúc cặp đĩa khi tương tác với sóng điện từ. Dễ dàng có thể nhận thấy rằng, kết quả mô phỏng rất trùng khớp với kết quả thực nghiệm và đều có một đỉnh không truyền qua ở tần số $14,2 \mathrm{GHz}$. Để khẳng định vùng không truyền qua này là cộng hưởng từ cho độ từ thẩm âm, chúng tôi đã nối tắt hai đầu cặp đĩa (connected dish pair - CDP) để loại bỏ tụ điện $(\mathrm{C})$. Kết quả cho thấy vùng không truyền qua do cộng hưởng trong cấu trúc DP được thay bằng đường truyền qua trong cấu trúc nối tắt (Hình 2(a)). Theo các kết quả nghiên cứu trước đây [9], có thể khẳng định vùng không truyền qua này của $\mathrm{DP}$ là do cộng hưởng từ. Mặt khác, kết quả tính toán độ từ thẩm thông qua các giá trị mô phỏng đối với cấu trúc $\mathrm{DP}$ và CDP đưa ra trên Hình 2(b) đã khẳng định thêm đây là cộng hưởng từ và cho độ từ thẩm âm tại vị trí cộng hưởng này.

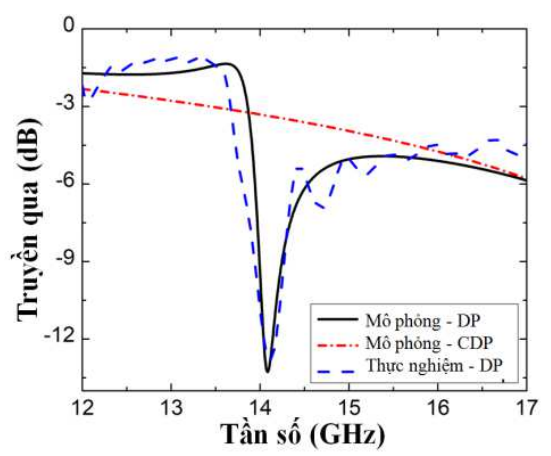

(a)

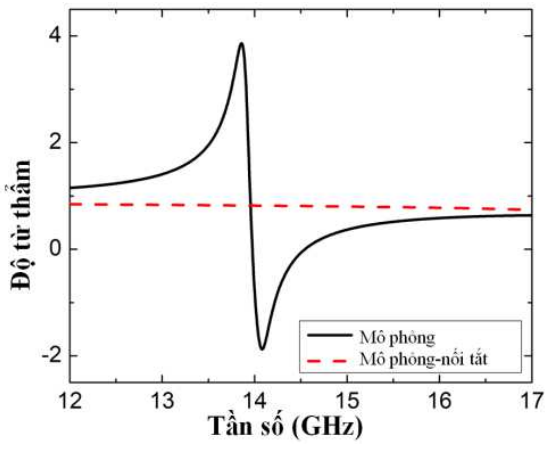

(b)

Hình 2. a) Phổ truyền qua thực nghiệm, mô phỏng của cấu trúc DP và cấu trúc DP bị nối tắt.

b) Phần thực độ từ thẩm của cấu trúc DP và DP bị nối tắt tính toán từ các số liệu mô phỏng.

Vùng độ từ thẩm âm của MMs thường được miêu tả bằng mô hình mạch LC. Sự tương tác giữa cộng hưởng $\mathrm{LC}$ với từ trường ngoài gây ra cộng hưởng từ. Theo các tài liệu nghiên cứu [10 - 12], một cách biến đổi và tính toán tương tự, tần số cộng hưởng từ của cấu trúc DP được biểu diễn bằng công thức:

$$
\mathrm{f}_{\mathrm{m}}=\frac{1}{2 \pi \sqrt{\mathrm{L}_{\mathrm{m}} \mathrm{C}_{\mathrm{m}}}}=\frac{\sqrt{t_{d}}}{\pi^{2} R \sqrt{\mathrm{c}_{1} \varepsilon \mu\left(t_{d}+2 t_{m}\right)}}
$$

Công thức (1.1) cho thấy, tần số cộng hưởng từ phụ thuộc vào các tham số cấu trúc như chiều dày lớp điện môi $t_{d}$ và kim loại $t_{m}$, hằng số điện mồi $\varepsilon$, đặc biệt phụ thuộc mạnh hơn vào bán kính $R$ của đĩa. $\mathrm{c}_{1}$ là tỉ số diện tích có điện tích phân bố trên diện tích toàn bộ của đĩa. Các kết quả khảo sát dựa trên mô phỏng và tính toán (dựa theo công thức 1.1) sự phụ thuộc của tần số này vào bán kính $\mathrm{R}$ được đưa ra trên Hình $3(\mathrm{~b})$. Các kết quả mô phỏng khá trùng khớp với các 
kết quả tính toán và đều cho thấy tần số cộng hưởng từ giảm khi bán kính $R$ tăng. Kết quả này gợi ý rằng, bằng cách điều khiển các tham số cấu trúc, đặc biệt là bán kính $\mathrm{R}$ ta có thể thu được các vùng có độ từ thẩm âm mong muốn.

Ngoài ra, kết quả nghiên cứu trên phổ truyền qua của cấu trúc DP phụ thuộc vào $R$ trên Hình 3(a) còn cho thấy khi bán kính $\mathrm{R}$ tăng từ $3 \mathrm{~mm}$ đến $4,05 \mathrm{~mm}$, tại vị trí $R=4,05 \mathrm{~mm}$ đường truyền qua có dáng điệu khác hẳn so với các đường còn lại. Cụ thể là xuất hiện một vùng truyền qua quanh tần số $15,3 \mathrm{GHz}$. Điều này sẽ được chúng tôi giải thích rõ ràng trong nghiên cứu tiếp theo và trình bày trong mục 3.2 .

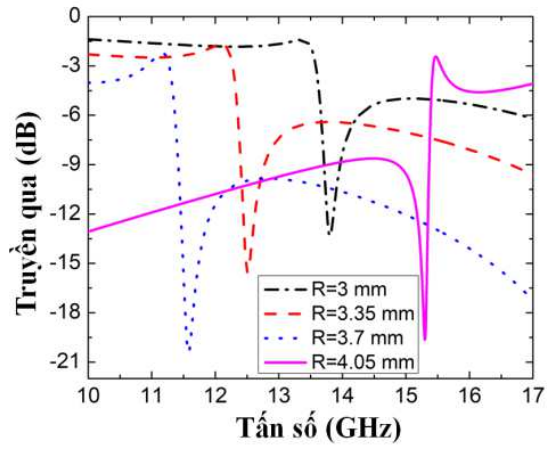

a)

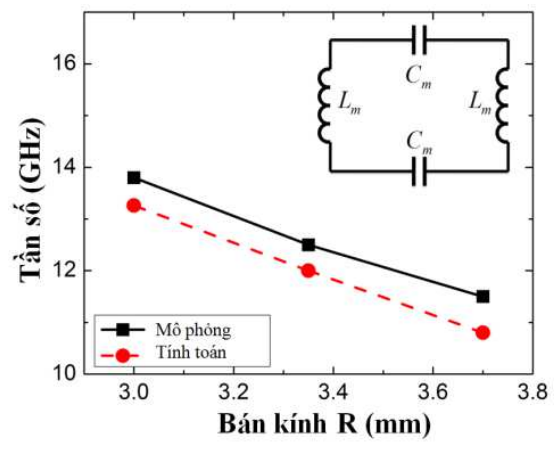

b)

Hình 3. Sự phụ thuộc a) phổ truyền qua và b) tần số cộng hưởng từ qua mô phỏng và tính toán vào bán kính $\mathrm{R}$ của đĩa trong cấu trúc cặp đĩa $a_{x}=11 \mathrm{~mm}, a_{y}=8 \mathrm{~mm}$ (hình nhỏ nằm trong hình $3(\mathrm{~b})$ là mô hình mạch điện LC của cấu trúc DP).

\subsection{Các kết quả nghiên cứu cấu trúc lưới đĩa (dish - net - DN)}

Để tìm hiểu được nguyên nhân của sự khác biệt của đường truyền qua tại vị trí $R=4,05$ $\mathrm{mm}$ như nói ở trên, trước tiên chúng ta đi tìm hiểu sự thay đổi về mặt cấu trúc tại vị trí $R$ này. Dễ dàng nhận thấy rằng, khi $R=4,05 \mathrm{~mm}$ thì hai hình tròn liên tiếp sẽ chạm vào nhau theo phương $\mathbf{E}$ của điện trường. Từ các phần tử từ $\mathrm{DP}$ tạo ra độ từ thẩm âm (hình 1(a) khi $R$ thay đổi từ $3 \mathrm{~mm}$ đến $3,7 \mathrm{~mm}$ ) sẽ được chuyển thành cấu trúc lưới đĩa $\mathrm{DN}$ (Hình 2(a) khi $R=4,05 \mathrm{~mm}$ ). Các hình tròn chạm vào nhau trong cấu trúc $\mathrm{DN}$ sẽ tạo ra các dây kim loại liên tục dọc theo trục $\mathrm{y}(\mathbf{E})$ và cho vùng có độ điện thẩm âm dưới tần số plasma khi tương tác với thành phần điện trường $\mathbf{E}$ của sóng điện từ chiếu tới. Vùng độ điện thẩm âm này kết hợp với vùng độ từ thẩm âm do thành phần từ DP cung cấp, kết quả là thu được một vùng chiết suất âm. Các kết quả tính toán các tham số hiệu dụng: độ điện thẩm, từ thẩm và chiết suất của cấu trúc $\mathrm{DN}$ này được đưa ra trên Hình 4(b). Hình 4(b) cho thấy, các kết quả tính toán hoàn toàn phù hợp với các nhận định trên, tồn tại một vùng quanh tần số $15,3 \mathrm{GHz}$ đồng thời có độ từ thẩm âm và điện thẩm âm nên chiết suất thu được là âm với độ tổn hao thấp [12]. Điều này giải thích cho việc quan sát thấy một vùng truyền qua có đỉnh ở $15,3 \mathrm{GHz}$ trong phổ truyền qua của cấu trúc $\mathrm{DN}(R=4,05 \mathrm{~mm})$ như đã nói ở trên. Kết quả mô phỏng và thực nghiệm của cấu trúc $\mathrm{DN}$ này được đưa ra trong Hình 4(a) cho thấy, số liệu thực nghiệm khá trùng khớp với mô phỏng, chỉ có một chút sai khác ở vị trí đỉnh truyền qua và được giải thích là do sai số trong quá trình chế tạo, đo đạc. 


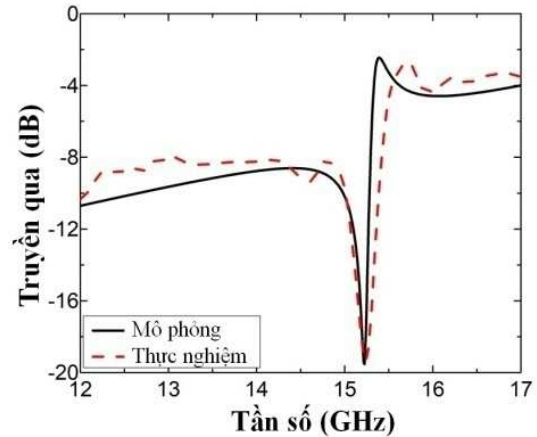

a)

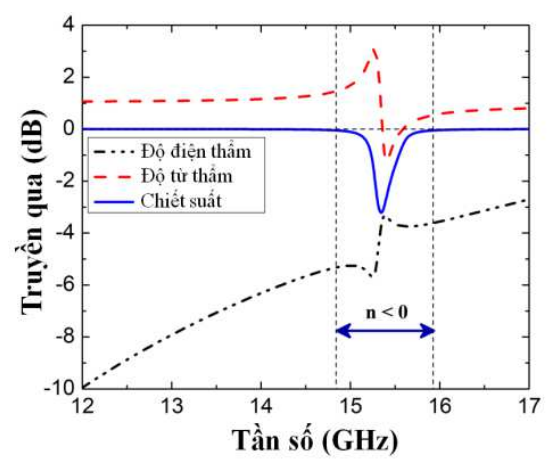

b)

Hình 4. a) Phổ truyền qua thực nghiệm và mô phỏng và b) Phần thực của độ điện thẩm, độ từ thẩm và chiết suất tính toán qua số liệu mô phỏng của cấu trúc DN có $a_{x}=11 \mathrm{~mm}, a_{y}=8 \mathrm{~mm}, R=4,05 \mathrm{~mm}$.

Một điều rất thú vị có thể nhận thấy qua các kết quả nghiên cứu ảnh hưởng của bán kính $R$ trong cấu trúc DP này là khi giá trị $R$ nhỏ thu được một vùng có độ từ thẩm âm, nhưng khi $R$ lớn đến mức hai đĩa liên tiếp chạm vào nhau thì vùng chiết suất âm được tạo ra. Điều này cho ta một gợi ý rằng, nếu ta thay thế đồng bằng một vật dẫn có hệ số dãn nở phụ thuộc mạnh vào tác động ngoại vi nào đó (như nhiệt, điện, quang..), thì tác dụng ngoại vi này có thể được sử dụng để chuyển đổi hai trạng thái - từ thẩm âm và chiết suất âm trong cấu trúc, khi đó ta có thể điều khiển được tính chất của vật liệu bằng tác động ngoại vi.

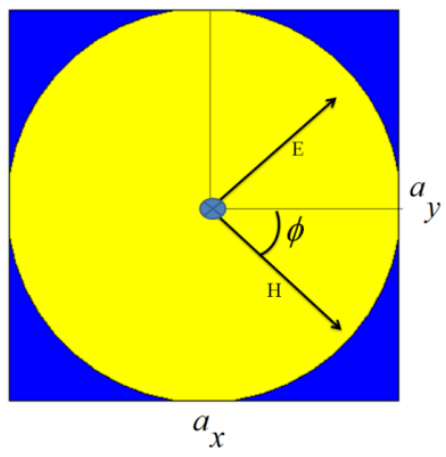

a)

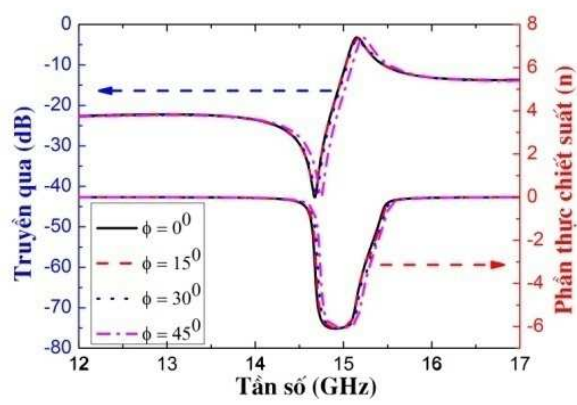

b)

Hình 5. a) Ô cơ sở của cấu trúc DN $a_{x}=a_{y}$ và góc quay phân cực. b) Phổ truyền qua mô phỏng của cấu trúc $\mathrm{DN} a_{x}=a_{y}=8 \mathrm{~mm}$ phụ thuộc vào góc phân cực.

Tuy nhiên, cấu trúc DN mà chúng tôi vừa khảo sát chưa tối ưu để đạt được chiết suất âm với mọi góc phân cực của sóng điện từ. Hằng số mạng theo các trục $a_{x}$ và $a_{y}$ khác nhau nên dễ dàng nhận thấy sự đối xứng chỉ thõa mãn với thành phần từ (các đĩa tròn), còn thành phần điện (các dây liên tục do các hình tròn tiếp xúc nhau tạo ra) chưa được thỏa mãn. Với mục đích tạo ra cấu trúc không phụ thuộc vào phân cực, thì cả hai thành phần này đều phải đối xứng với các thành phần của sóng điện từ. Điều này được thực hiện đơn giản khi cho các hằng số mạng bằng nhau $a_{x}=a_{y}$. Hình 5(a) là ô cơ sở của cấu trúc DN có $a_{x}=a_{y}$. Các kết quả nghiên cứu sự phụ thuộc vào phân cực sóng điện từ của phổ truyền qua và phần thực của chiết suất với mẫu $\mathrm{DN}$ có $a_{x}=a_{y}=8 \mathrm{~mm}$ được đưa ra trong Hình $5(\mathrm{~b})$. Kết quả cho thấy rằng, tính chất chiết suất âm đạt được quanh vùng $15 \mathrm{GHz}$ và phổ truyền qua của cấu trúc DN không thay đổi khi góc phân cực 
khi thay đổi từ $0^{0}$ đến $45^{0}$. Do tính chất đối xứng nên kết quả này sẽ giữ nguyên với mọi góc phân cực khi thay đổi các hướng của thành phần $\mathbf{E}$ và $\mathbf{H}$ của sóng điện từ chiếu tới.

\section{KẾT LUẬN}

Trong báo cáo này, chúng tôi đã sử dụng cấu trúc biến đổi của CWP - cấu trúc cặp đãa có tính đối xứng cao để tạo cộng hưởng từ cho độ từ thẩm âm mà không phụ thuộc vào phân cực của sóng điện từ chiếu đến. Sự phụ thuộc của tần số cộng hưởng từ vào bán kính của đĩa đã được mô phỏng và tính toán cho kết quả khá trùng khớp với nhau. Kết quả tần số cộng hưởng từ giảm khi bán kính tăng đã được giải thích bằng mô hình mạch điện LC. Bằng cách mở rộng bán kính của đĩa cho đến khi các đĩa liên tiếp chạm vào nhau, tính chiết suất âm thu được. Điều này đưa ra một gợi ý là có thể điều khiển tính chất của siêu vật liệu bằng các tác động ngoại vi. Cấu trúc tối ưu cho vùng chiết suất âm không phụ thuộc vào phân cực của sóng tới tìm kiếm được gồm các đĩa liên tiếp chạm vào nhau dọc theo cả hai trục $\mathrm{x}$ và $\mathrm{y}$. Kết quả này là một bước quan trọng để tiến gần đến các ứng dụng thực của hiện tượng chiết suất âm khi không phụ thuộc phân cực.

Lòi cảm ơn. Công trình này được thực hiện với sự hỗ trợ của đề tài Khoa học và Công nghệ Bộ giáo dục và đào tạo B2015-TN05-01 và đề tài cấp Viện Hàn lâm Khoa học và Công nghệ Việt Nam mã số 103.022013.54.

\section{TÀI LIỆU THAM KHẢO}

1. Ashcroft N. W., Mermin N. D. - Solid State Physics, (Saunders College, Philadelphia, 1976), There No Corresp. Rec. This Ref. (1981) 673.

2. Khanna S. N., Jena P. - Assembling crystals from clusters, Phys. Rev. Lett. 69 (1992) 1664.

3. Veselago V. G. - The electrodynamics of substances with simultaneously negative values of $\varepsilon$ and $\mu$, Sov. Phys. Usp. 10 (1968) 509.

4. Pendry J., Holden A., Robbins D., and Stewart W. - Magnetism from conductors and enhanced nonlinear phenomena, Microwave Theory and Techniques, IEEE Transactions on, 47 (1999) 2075.

5. Smith D., Padilla W., Vier D., Nemat-Nesser S. and Chultz S. - Composite medium with simultaneously negative permeability and permittivity, Phys. Rev. Lett. 84 (2000) 4184.

6. Chen C. C., IshikawaA., TangY. H., ShiaoM. H., Tsai D. P. and Tanaka T. - Uniaxialisotropic Metamaterials by Three-Dimensional Split-Ring Resonators, Adv. Opt. Mat. 3 (2015) 138.

7. Viet D. T., Hieu N. V., Lam V. D. and Tung N. T. - Isotropic metamaterial absorber using cut-wire-pair structures, Appl. Phys. Express 8 (2015) 032001.

8. Chen X., Grzegorczyk T. M., Wu B. I., Pacheco J., and Kong J. A. - Robust method to retrieve the constitutive effective parameters of metamaterials, Phys. Rev. E. 70 (2004) 016608

9. Tung N. T., Lievens P., Lee Y. P. and Lam V. D. - Computational studies of a cut-wire pair and combined metamaterials, Adv. Nat. Sci.: Nanosci. Nanotechnol. 2 (2011) 033001.

10. Zhou J., Economon E. N., Koschny T., and Soukoulis C. M. - Unifying approach to lefthanded material design, Opt. Lett. 31 (2006) 3620.

11. Hien N. T., Le L. N., Trang P. T., Tung B. S., Viet D. T., Lee Y. P., Lam V. D., Tung N. 
T. - Characterizations of a thermo-tunable broadband fishnet metamaterial at $\mathrm{THz}$ frequencies, Com. Mat. Sci.103 (2015) 189.

12. Tung B. S., Tung N. T., Lam V. D - Thermally tunable magnetic metamaterials at THz frequencies, J. Opt. 15 (2013) 075101.

13. Tung N. T, Peter L., Lee Y. P., Lam V. D. - Computational studies of a cut-wire pair and combined metamaterials, Adv. Nat. Sci.: Nanosci. Nanotechnol. 2 (2011) 033001.

\title{
ABSTRACT \\ STUDY, DESIGN AND FABRICATION OF POLARIZATION-INDEPENDENT METAMATERIALS
}

\author{
Nguyen Thi Hien ${ }^{1,2, *}$, Vu Đinh Qui ${ }^{1}$, Trinh Thi Giang ${ }^{1}$, Nguyen Thanh Tung ${ }^{1}$, Vu Đinh Lam ${ }^{1}$ \\ ${ }^{1}$ Institute of Materials Science, VAST, 18 Hoang Quoc Viet, Cau Giay, Hanoi \\ ${ }^{2}$ Thai Nguyen University of Sciences, Tan Thinh Ward, Thai Nguyen city \\ "Email: ncshien@gmail.com
}

Conventionally, to obtain the negative permeability and negative refraction metamaterials, the split-ring resonator (SRR) and cut-wire-pair (CWP) were applied. However, the properties of these structuresare strongly dependent on the polarization of electromagnetic waves. In this report, we used a high symmetry structure -the so-called dish pair structurewhich based on the CWP structure, with the aim of removing this drawback. Each unit cell consists of two identical dishes, symmetrically arranged in a dielectric spacer, providing the negative permeability when interacting with the electromagnetic wave. By expanding the radius of the dish until two adjacent dish close each other, the negative refractive index can be obtained. This is important effect to have a tunable MMs by interacting with the external field. The results of studies using the simulation, experimental and calculation quite coincide. The obtained structuredoes not depend on the polarization of the incident wave. This result is very important for practical applications using the isotropic negative refraction metamaterials.

Keywords: isotropic negative refraction metamaterial, dish pair, dish net. 